\title{
Time-Varying Linearization and the Perron effects
}

\author{
Leonov G.A., Kuznetsov N.V.
}

\author{
Draft $^{1} 2$
}

Keywords: Lyapunov exponent; Lyapunov exponents sign inversion; Characteristic exponent; Lyapunov characteristic exponent; chaos; time-varying linearization; nonstationary; stability by the first approximation; instability; Perron effects; counterexample; strange attractor

\section{Introduction: negative largest Lyapunov exponent doesn't, in general, indicate stability and positive largest Lyapunov expo- nent doesn't, in general, indicate chaos}

In 1892, the general problem of stability by the first approximation was considered by Lyapunov. He proved that if the system of the first approximation is regular and all its Lyapunov exponents (or characteristic exponents) are negative, then the solution of the original system is asymptotically Lyapunov stable. In 1930, it was stated by $\mathrm{O}$. Perron that the requirement of regularity of the first approximation is substantial. He constructed an example of the second-order system of the first approximation, which has negative characteristic exponents along a zero solution of the original system but, at the same time, this zero solution of original system is Lyapunov unstable. Furthermore, in a certain neighborhood of this zero solution almost all solutions of original system have positive characteristic exponents. The effect of a sign reversal of characteristic exponents of solutions of the original system and the system of first approximation with the same initial data was subsequently called the Perron effect.

So positive largest Lyapunov exponent doesn't, in general, indicate chaos and negative largest Lyapunov exponent doesn't, in general, indicate stability!

The counterexample of Perron impressed on the contemporaries and gave an idea of the difficulties arising in the justification of the first approximation theory for nonautonomous and nonperiodic linearizations. Later, Persidskii [1947], Massera [1957], Malkin [1966], and Chetaev [1955], obtained sufficient conditions of stability by the first approximation for nonregular linearizations generalizing the Lyapunov theorem. At the same time, according to Malkin (Theory of Motion Stability, 1966):

"... The counterexample of Perron shows that the negativeness of Lyapunov exponents (or characteristic exponents) is not a sufficient condition of stability by the first approximation. In the general case necessary and sufficient conditions of stability by the first approximation are not obtained."

For certain situations, the results on stability by the first approximation can be found in the books |Bellman \& Cooke, 1963, Davies \& James, 1966, Willems, 1970, Coppel, 1965, Wasow, 1965, Bellman, 1953, Bacciotti \& Rosier, 2005, Yoshizawa, 1966, Lakshmikantham et al., 1989, Hartman, 1984, Halanay, 1966, Sansone 8

In the 1940s Chetaev [1948] published the criterion of instability by the first approximation for regular linearizations. However, in the proof of these criteria a flaw was discovered and, at present, the complete proof of Chetaev theorems is given for a more weak condition in comparison with that for instability by Lyapunov, namely, for instability by Krasovsky only. The discovery of strange attractors was made obvious with the study of instability by the first approximation. Nowadays the problem of the justification of the nonstationary

\footnotetext{
${ }^{1}$ Nikolay V. Kuznetsov, nkuznetsov239 at gmail.com (correspondence author)

${ }^{2}$ PDF slides http://www.math.spbu.ru/user/nk/PDF/Lyapunov-exponent-Sign-inversion-Perron-effects-Chaos.pdf
} 
linearizations for complicated nonperiodic motions on strange attractors bears a striking resemblance to the situation that occurred 120 years ago. The founders of the automatic control theory J. Maxwell [1868], and A.I. Vyschegradskii [1877] courageously used a linearization in a neighborhood of stationary motions, leaving the justification of such linearization to A. Poincare [1886] and A.M. Lyapunov [1892]. At present, many specialists in chaotic dynamics believe that the positiveness of the largest characteristic exponent of a linear system of the first approximation implies the instability of solutions of the original system. Moreover, there are a number of computer experiments, in which the various numerical methods for calculating the characteristic exponents and the Lyapunov exponents of linear systems of the first approximation are used. As a rule, authors ignore the justification of the linearization procedure and use the numerical values of exponents so obtained to construct various numerical characteristics of attractors of the original nonlinear systems (Lyapunov dimensions, metric entropies, and so on). Sometimes, computer experiments serve as arguments for the partial justification of the linearization procedure.

So, the approach based on linearizations along the trajectories on the strange attractors require justification. This motivates to the development of the nonstationary theory of instability by the first approximation.

This chapter shows the contemporary state of the art of the problem of the justification of nonstationary linearizations. Here for the discrete and continuous systems the results of stability by the first approximation for regular and nonregular linearizations are given, the Perron effects are considered, the criteria of the stability and instability of the flow and cascade of solutions and the criteria of instability by Lyapunov and Krasovsky are obtained.

\section{Characteristic Exponents, Regular Systems, Lyapunov Expo- nents}

Consider systems with a marked linear part. In the continuous case we have

$$
\frac{d x}{d t}=A(t) x+f(t, x), x \in \mathbb{R}^{n}, \quad t \in[0,+\infty),
$$

where $A(t)$ is a continuous $(n \times n)$-matrix, $f(\cdot, \cdot):[0,+\infty) \times \mathbb{R}^{n} \rightarrow \mathbb{R}^{n}$ is a continuous vector-function. Suppose,

$$
\sup _{t \in[0,+\infty)}|A(t)|<+\infty
$$

in the continuous case.

In the discrete case, we have

$$
x(t+1)=A(t) x(t)+f(t, x(t)), x(t) \in \mathbb{R}^{n}, \quad t \in \mathbb{N}_{0},
$$

where $A(t)$ is an $(n \times n)$-matrix, $f(\cdot, \cdot): \mathbb{N}_{0} \times \mathbb{R}^{n} \rightarrow \mathbb{R}^{n}$. In this case, we assume that

$$
\operatorname{det} A(t) \neq 0, \quad \sup _{t \in \mathbb{N}_{0}}|A(t)|<+\infty, \quad \sup _{t \in \mathbb{N}_{0}}\left|A(t)^{-1}\right|<+\infty .
$$

Here $|\cdot|$ is the Euclidean norm.

Suppose, in a certain neighborhood $\Omega(0)$ of the point $x=0$ the nonlinear parts of systems (1) and (1') satisfy the following condition

$$
|f(t, x)| \leq \kappa|x|^{\nu} \quad \forall t \geq 0, \quad \forall x \in \Omega(0), \quad \kappa>0, \nu>1
$$

We shall say that the first approximation system for (1) is the following linear system

$$
\frac{d x}{d t}=A(t) x
$$


and that for discrete system (1') is the linear system

$$
x(t+1)=A(t) x(t) .
$$

Consider a fundamental matrix $X(t)=\left(x_{1}(t), \ldots, x_{n}(t)\right)$, consisting of the linear-independent solutions $\left\{x_{i}(t)\right\}_{1}^{n}$ of the first approximation system. For the determinant of the fundamental matrix we have the Ostrogradsky-Liouville formula, which in the continuous case is as follows

$$
\operatorname{det} X(t)=\operatorname{det} X(0) \exp \left(\int_{0}^{t} \operatorname{Tr} A(\tau) d \tau\right),
$$

and in the discrete one takes the form

$$
\operatorname{det} X(t)=\operatorname{det} X(0) \prod_{j=0}^{t-1} \operatorname{det} A(j) .
$$

The fundamental matrices are often considered to satisfy the following condition

$$
X(0)=I_{n}
$$

where $I_{n}$ is a unit $(n \times n)$-matrix.

These definitions and results are valid for continuous system as well as for the discrete one. The proofs will be given, if necessary, for each situation separately.

Consider the vector-function $f(t)$ such that $\lim _{t \rightarrow+\infty} \sup |f(t)| \neq 0$.

Definition 1 The value (or the symbol $+\infty$, or $-\infty$ ), defined by formula

$$
\mathcal{X}[f(t)]=\lim _{t \rightarrow+\infty} \sup \frac{1}{t} \ln |f(t)|,
$$

is called a characteristic exponent of the vector-function $f(t)$.

The characteristic exponent is equal to that taken with inverse sign characteristic number, introduced by Lyapunov [1892].

Definition 2 The characteristic exponent of the vector-function $f(t)$ is said to be exact if the finite limit

$$
\mathcal{X}[f(t)]=\lim _{t \rightarrow+\infty} \frac{1}{t} \ln |f(t)|
$$

exists.

Consider the characteristic exponents of solutions of linear system (3) or (3').

Definition 3 [Demidovich, 1967 ' A set of distinctive characteristic exponents of all solutions (except a zero solution), being different from $\pm \infty$, of linear system is called its spectrum.

Note that the number of different characteristic exponents is bounded by the dimension of the considered space of system states. 


\section{$2.1 \quad$ Regular systems}

Consider the normal fundamental systems of solutions introduced by Lyapunov [1892].

Definition 4 A fundamental matrix is said to be normal if the sum of characteristic exponents of its columns is minimal in comparison with other fundamental matrices.

Definition 5 [Demidovich, 1967] The set of characteristic exponents

$$
\lambda_{1}, \ldots, \lambda_{n}
$$

of a certain normal fundamental system of solutions is called a complete spectrum and the number $\sigma=\sum_{1}^{n} \lambda_{i}$ is a sum of characteristic exponents of linear system.

Definition 6 A linear system is said to be regular if for the sum of its characteristic exponents $\sigma$ the following relation holds

$$
\sigma=\lim _{t \rightarrow+\infty} \inf \frac{1}{t} \ln |\operatorname{det} X(t)|
$$

Taking into account formula (4), in the continuous case we obtain a classical definition |Demidovich, 1967, Andrianova, 1998| of the regularity of system

$$
\sigma=\lim _{t \rightarrow+\infty} \inf \frac{1}{t} \int_{0}^{t} \operatorname{Tr} A(\tau) \mathrm{d} \tau .
$$

Similarly, formula (4') gives a definition of regularity |Demidovich, 1969| in the discrete case

$$
\sigma=\lim _{t \rightarrow+\infty} \inf \frac{1}{t} \ln \prod_{j=0}^{t-1}|\operatorname{det} A(j)| .
$$

Definition 7 The number

$$
\Gamma=\sigma-\lim _{t \rightarrow+\infty} \inf \frac{1}{t} \ln |\operatorname{det} X(t)|
$$

is called an irregularity coefficient of linear system.

As was shown in [Demidovich, 1967|, the systems with constant and periodic coefficients are regular.

For continuous |Demidovich, 1967| and discrete systems |Demidovich, 1969, Gayschun, 2001| the following is well known

Lemma 1 (Lyapunov inequality)

Let all characteristic exponents of solutions of linear system be $<+\infty$ ( or all characteristic exponents be $>-\infty$.)

Then, for any fundamental system of solutions $X(t)$ the following inequality

$$
\lim _{t \rightarrow+\infty} \sup \frac{1}{t} \ln |\operatorname{det} X(t)| \leq \sigma_{X}
$$

where $\sigma_{X}$ is a sum of characteristic exponents of the system of solutions $X(t)$, is satisfied. 
Thus, for regular systems there exists the limit

$$
\lim _{t \rightarrow+\infty} \frac{1}{t} \ln |\operatorname{det} X(t)| \text {. }
$$

Note that from the condition of regularity of linear system it follows |Demidovich, 1967| that for its solutions $x(t) \neq 0$ there exist the limits

$$
\lim _{t \rightarrow+\infty} \frac{1}{t} \ln |x(t)| \text {. }
$$

Note 1 As was shown in 'Bylov et al., 1966], the opposite, generally speaking, is not valid. We give an example of nonregular system, all characteristic exponents of which are exact 'Bylov et al., 1966].

Consider system (3) with the matrix

$$
A(t)=\left(\begin{array}{cc}
0 & 1 \\
0 & (\cos \ln t-\sin \ln t-1)
\end{array}\right), \quad t \geq 1
$$

and its fundamental matrix $X(t)$

$$
X(t)=\left(x_{1}(t), x_{2}(t)\right)=\left(\begin{array}{ccc} 
& t & \int^{\gamma(\tau)} \mathrm{d} \tau \\
& 1 & e^{\gamma(t)}
\end{array}\right),
$$

where $\gamma(t)=t(\cos \ln t-1)$. In this case for the determinant of fundamental matrix the following relation

$$
\lim _{t \rightarrow+\infty} \inf \frac{1}{t} \ln |\operatorname{det} X(t)|=-2
$$

is satisfied. We now find characteristic exponents of solutions. For $x_{1}(t)$ we have

$$
\lim _{t \rightarrow+\infty} \sup \frac{1}{t} \ln \left|x_{1}(t)\right|=\lim _{t \rightarrow+\infty} \inf \frac{1}{t} \ln \left|x_{1}(t)\right|=0 .
$$

Since $e^{\gamma(t)} \leq 1$ for $t \geq 1$, we conclude that the characteristic exponent $x_{2}(t)$ is less than or equal to zero

$$
\lim _{t \rightarrow+\infty} \sup \frac{1}{t} \ln \left|x_{2}(t)\right| \leq 0
$$

On the other hand, since the integral of $e^{\gamma(\tau)}$ is divergent, namely

$$
\int_{1}^{+\infty} e^{\gamma(\tau)} \mathrm{d} \tau=+\infty
$$

for $x_{2}(t)$ we have the following estimate

$$
\lim _{t \rightarrow+\infty} \inf \frac{1}{t} \ln \left|x_{2}(t)\right| \geq 0
$$

This implies that

$$
\lim _{t \rightarrow+\infty} \sup \frac{1}{t} \ln \left|x_{2}(t)\right|=\lim _{t \rightarrow+\infty} \inf \frac{1}{t} \ln \left|x_{2}(t)\right|=0 .
$$

Thus, by (7), (8), and (10) the linear system with matrix (6) has exact characteristic exponents but it is nonregular:

$$
\Gamma=2 .
$$




\subsection{Lyapunov exponents and singular values}

Consider singular values of the matrix $X(t) \mid$ Hahn, 1967, Katok \& Hasselblat, 1995, Boichenko et al., 2005|.

Definition 8 The singular values $\left\{\alpha_{j}(X(t))\right\}_{1}^{n}$ of the matrix $X(t)$ are the square roots of the eigenvalues of the matrix $X(t)^{*} X(t)$.

The following geometric interpretation of singular values is known: the numbers $\alpha_{j}(X(t))$ coincide with a principal semiaxis of the ellipsoid $X(t) B$, where $B$ is a ball of unit radius.

Definition 9 'Temam, 1988$]$ The Lyapunov exponent $\mu_{j}$ is as follows

$$
\mu_{j}=\lim _{t \rightarrow+\infty} \sup \frac{1}{t} \ln \alpha_{j}(X(t)) .
$$

Let $\mu_{1}$ and $\lambda_{1}$ be the largest Lyapunov exponent and the largest characteristic exponent, respectively.

Lemma 2 For the linear systems the largest characteristic exponent is equal to the largest Lyapunov exponent.

Note 2 We will show that there exist systems such that the characteristic exponents do not coincide with the Lyapunov exponents

We give an example |Leonov, 2008| of such system in the continuous case. Consider system (3) with the matrix

$$
A(t)=\left(\begin{array}{cc}
0 & \sin (\ln t)+\cos (\ln t) \\
\sin (\ln t)+\cos (\ln t) & 0
\end{array}\right) \quad t>1
$$

and with the fundamental normal matrix

$$
X(t)=\left(\begin{array}{cc}
e^{\gamma(t)} & e^{-\gamma(t)} \\
e^{\gamma(t)} & -e^{-\gamma(t)}
\end{array}\right),
$$

where $\gamma(t)=t \sin (\ln t)$. It is obvious that $\lambda_{1}=\lambda_{2}=1$ and

$$
\begin{aligned}
& \alpha_{1}(X(t))=\sqrt{2} \max \left(e^{\gamma(t)}, e^{-\gamma(t)}\right) \\
& \alpha_{2}(X(t))=\sqrt{2} \min \left(e^{\gamma(t)}, e^{-\gamma(t)}\right) .
\end{aligned}
$$

This implies the following relations $\mu_{1}=1, \mu_{2}=0$. Thus, we have $\lambda_{2} \neq \mu_{2}$.

Lemma 3 A linear system is regular if and only if

$$
\lim _{t \rightarrow+\infty} \inf \frac{1}{t} \ln \alpha_{j}(X(t))=\lim _{t \rightarrow+\infty} \sup \frac{1}{t} \ln \alpha_{j}(X(t)), \quad j=1, . ., n .
$$




\subsection{The Nemytskii - Vinograd counterexample}

Consider a continuous system |Bylov et al., 1966|

$$
\frac{d x}{d t}=A(t) x
$$

with the matrix

$$
A(t)=\left(\begin{array}{cc}
1-4(\cos 2 t)^{2} & 2+2 \sin 4 t \\
-2+2 \sin 4 t & 1-4(\sin 2 t)^{2}
\end{array}\right) .
$$

In this case, its solution is the vector-function

$$
x(t)=\left(\begin{array}{c}
e^{t} \sin 2 t \\
e^{t} \cos 2 t
\end{array}\right) .
$$

It follows that

$$
\operatorname{det}\left(A(t)-p I_{n}\right)=p^{2}+2 p+1
$$

Therefore for the eigenvalues $\nu_{1}(t)$ and $\nu_{2}(t)$ of the matrix $A(t)$ we have

$$
\nu_{1}(t)=\nu_{2}(t)=-1 .
$$

On the other hand, the characteristic exponent $\lambda$ of solution (13) is equal to 1 .

This counterexample shows that all eigenvalues of the matrix $A(t)$ can have negative real parts even if the corresponding linear system has positive characteristic exponents.

It also shows that the formula, obtained in the book |Anishchenko et al., 2002|, namely

$$
\lambda_{j}=\limsup _{t \rightarrow+\infty} \frac{1}{t} \int_{0}^{t} \operatorname{Re} \nu_{j}(\tau) d \tau
$$

is untrue.

\section{The Perron Effects}

In 1930, O. Perron |Perron, 1930| showed that the negativeness of the largest characteristic exponent of the first approximation system does not always result in the stability of zero solution of the original system. Furthermore, in an arbitrary small neighborhood of zero, the solutions of the original system with positive characteristic exponent can be found. These results of Perron impressed on the specialists in the theory of stability of motion.

The effect of sign reversal of characteristic exponent of the solutions of the first approximation system and of the original system with the same initial data we shall call |Leonov, 1998, Leonov, 2004| the Perron effect.

We now present the outstanding result of Perron [1930] and its discrete analog |Kuznetsov \& Leonov, 2001, Gayschun, 2001|.

Consider the following system

$$
\begin{aligned}
& \frac{d x_{1}}{d t}=-a x_{1} \\
& \frac{d x_{2}}{d t}=(\sin (\ln (t+1))+\cos (\ln (t+1))-2 a) x_{2}+x_{1}^{2}
\end{aligned}
$$


and its discrete analog

$$
\begin{aligned}
& x_{1}(t+1)=\exp (-a) x_{1}(t) \\
& x_{2}(t+1)=\frac{\exp ((t+2) \sin \ln (t+2)-2 a(t+1))}{\exp ((t+1) \sin \ln (t+1)-2 a t)} x_{2}(t)+x_{1}(t)^{2} .
\end{aligned}
$$

Here $a$ is a number satisfying the following inequalities

$$
1<2 a<1+\frac{1}{2} \exp (-\pi)
$$

The solution of the first approximation system for systems (14) and (14') takes the form

$$
\begin{aligned}
& x_{1}(t)=\exp (-a t) x_{1}(0) \\
& x_{2}(t)=\exp ((t+1) \sin (\ln (t+1))-2 a t) x_{2}(0) .
\end{aligned}
$$

It is obvious that by condition (15) for the solution of the first approximation system for $x_{1}(0) \neq 0, x_{2}(0) \neq 0$ we have

$$
\mathcal{X}\left[x_{1}(t)\right]=-a, \mathcal{X}\left[x_{2}(t)\right]=1-2 a<0 .
$$

This implies that a zero solution of linear system of the first approximation is Lyapunov stable.

Now we consider the solution of system (14)

$$
\begin{aligned}
& x_{1}(t)=\exp (-a t) x_{1}(0) \\
& x_{2}(t)=\exp ((t+1) \sin (\ln (t+1))-2 a t) \times \\
& \times\left(x_{2}(0)+x_{1}(0)^{2} \int_{0}^{t} \exp (-(\tau+1) \sin (\ln (\tau+1))) d \tau\right) .
\end{aligned}
$$

Assuming $t=t_{k}=\exp \left(\left(2 k+\frac{1}{2}\right) \pi\right)-1$, where $k$ is an integer, we obtain

$$
\begin{gathered}
\exp ((t+1) \sin (\ln (t+1))-2 a t)=\exp ((1-2 a) t+1), \quad(1+t) e^{-\pi}-1>0 \\
\int_{0}^{t} \exp (-(\tau+1) \sin (\ln (\tau+1))) d \tau> \\
>\int_{f(k)}^{g(k)} \exp (-(\tau+1) \sin (\ln (\tau+1))) d \tau> \\
>\int_{f(k)}^{g(k)} \exp \left(\frac{1}{2}(\tau+1)\right) d \tau>\int_{f(k)}^{g(k)} \exp \left(\frac{1}{2}(\tau+1) \exp (-\pi)\right) d \tau= \\
=\exp \left(\frac{1}{2}(t+1) \exp (-\pi)\right)(t+1)\left(\exp \left(-\frac{2 \pi}{3}\right)-\exp (-\pi)\right)
\end{gathered}
$$

where

$$
\begin{gathered}
f(k)=(1+t) \exp (-\pi)-1 \\
g(k)=(1+t) \exp \left(-\frac{2 \pi}{3}\right)-1
\end{gathered}
$$


Hence we have the following estimate

$$
\begin{aligned}
& \exp ((t+1) \sin (\ln (t+1))-2 a t) \int_{0}^{t} \exp (-(\tau+1) \sin (\ln (\tau+1))) d \tau> \\
& >\exp \left(\frac{1}{2}(2+\exp (-\pi))\left(\exp \left(-\frac{2 \pi}{3}\right)-\exp (-\pi)\right)(t+1) \times\right. \\
& \quad \times \exp \left(\left(1-2 a+\frac{1}{2} \exp (-\pi)\right) t\right) .
\end{aligned}
$$

From the last inequality and condition (15) it follows that for $x_{1}(0) \neq 0$ one of the characteristic exponents of solutions of system (14) is positive:

$$
\mathcal{X}\left[x_{1}(t)\right]=-a, \mathcal{X}\left[x_{2}(t)\right] \geq 1-2 a+e^{-\pi} / 2>0 .
$$

Thus, we obtain that all characteristic exponents of the first approximation system are negative but almost all solutions of the original system (14) tend exponentially to infinity as $t_{k} \rightarrow+\infty$.

Consider now the solution of discrete system (14')

$$
\begin{aligned}
x_{1}(t)= & x_{1}(0) e^{-a t} \\
x_{2}(t)= & \exp ((t+1) \sin \ln (t+1)-2 a t) \times \\
& \times\left(x_{2}(0)+x_{1}(0)^{2} \sum_{k=0}^{t-1} \exp (-(k+2) \sin \ln (k+2)+2 a)\right),
\end{aligned}
$$

and show that for this system inequalities (18) are also satisfied.

...

It follows that for $x_{1}(0) \neq 0$ one of characteristic exponents of solutions (19) of system (14') is positive and inequalities (18) are satisfied.

We give an example, which show the possibility of the sign reversal of characteristic exponents "on the contrary", namely the solution of the first approximation system has a positive characteristic exponent while the solution of the original system with the same initial data has a negative exponent.

Consider the following continuous system |Leonov, 2004|

$$
\begin{aligned}
& \dot{x}_{1}=-a x_{1} \\
& \dot{x}_{2}=-2 a x_{2} \\
& \dot{x}_{3}=(\sin (\ln (t+1))+\cos (\ln (t+1))-2 a) x_{3}+x_{2}-x_{1}^{2}
\end{aligned}
$$

and its discrete analog

$$
\begin{aligned}
& x_{1}(t+1)=e^{-a} x_{1}(t) \\
& x_{2}(t+1)=e^{-2 a} x_{2}(t) \\
& x_{3}(t+1)=\frac{\exp ((t+2) \sin \ln (t+2)-2 a(t+1))}{\exp ((t+1) \sin \ln (t+1)-2 a t)} x_{3}(t)+x_{2}(t)-x_{1}(t)^{2}
\end{aligned}
$$

on the invariant manifold

$$
M=\left\{x_{3} \in \mathbb{R}^{1}, x_{2}=x_{1}^{2}\right\} .
$$

Here the value $a$ satisfies condition (15). 
The solutions of $(20)$ and $\left(20^{\prime}\right)$ on the manifold $M$ take the form

$$
\begin{aligned}
& x_{1}(t)=\exp (-a t) x_{1}(0) \\
& x_{2}(t)=\exp (-2 a t) x_{2}(0) \\
& x_{3}(t)=\exp ((t+1) \sin (\ln (t+1))-2 a t) x_{3}(0), \\
& x_{1}(0)^{2}=x_{2}(0)
\end{aligned}
$$

Obviously, these solutions have negative characteristic exponents.

For system (20) in the neighborhood of its zero solution, consider the system of the first approximation

$$
\begin{aligned}
& \dot{x}_{1}=-a x_{1} \\
& \dot{x}_{2}=-2 a x_{2} \\
& \dot{x}_{3}=(\sin (\ln (t+1))+\cos (\ln (t+1))-2 a) x_{3}+x_{2} .
\end{aligned}
$$

The solutions of this system are the following

$$
\begin{aligned}
x_{1}(t) & =\exp (-a t) x_{1}(0) \\
x_{2}(t) & =\exp (-2 a t) x_{2}(0) \\
x_{3}(t) & =\exp ((t+1) \sin (\ln (t+1))-2 a t) \times \\
& \times\left(x_{3}(0)+x_{2}(0) \int_{0}^{t} \exp (-(\tau+1) \sin (\ln (\tau+1))) d \tau\right) .
\end{aligned}
$$

For system $\left(20^{\prime}\right)$ in the neighborhood of its zero solution, the system of the first approximation is as follows

$$
\begin{aligned}
& x_{1}(t+1)=\exp (-a) x_{1}(t) \\
& x_{2}(t+1)=\exp (-2 a) x_{2}(t) \\
& x_{3}(t+1)=\frac{\exp ((t+2) \sin \ln (t+2)-2 a(t+1))}{\exp ((t+1) \sin \ln (t+1)-2 a t)} x_{3}(t)+x_{2}(t) .
\end{aligned}
$$

Then the solutions of system (22') take the form

$$
\begin{aligned}
x_{1}(t) & =\exp (-a t) x_{1}(0) \\
x_{2}(t) & =\exp (-2 a t) x_{2}(0) \\
x_{3}(t) & =\exp ((t+1) \sin \ln (t+1)-2 a t) \times \\
& \times\left(x_{3}(0)+x_{2}(0)^{2} \sum_{k=0}^{t-1} \exp (-(k+2) \sin \ln (k+2)+2 a)\right) .
\end{aligned}
$$

By estimates (17) for solutions (23) and (23') for $x_{2}(0) \neq 0$ we obtain

$$
\mathcal{X}\left[x_{3}(t)\right]>0 .
$$

It is easily shown that for solutions of systems (20) and (22) the following relations

$$
\left(x_{1}(t)^{2}-x_{2}(t)\right)^{\bullet}=-2 a\left(x_{1}(t)^{2}-x_{2}(t)\right)
$$

are valid. Similarly, for system (22') we have

$$
x_{1}(t+1)^{2}-x_{2}(t+1)=\exp (-2 a)\left(x_{1}(t)^{2}-x_{2}(t)\right)
$$


Then

$$
x_{1}(t)^{2}-x_{2}(t)=\exp (-2 a t)\left(x_{1}(0)^{2}-x_{2}(0)\right) .
$$

It follows that the manifold $M$ is an invariant exponentially attractive manifold for solutions of continuous systems (20) and (22), and for solutions of discrete systems (20') and (22').

This means that the relation $x_{1}(0)^{2}=x_{2}(0)$ yields the relation $x_{1}(t)^{2}=x_{2}(t)$ for all $t \in \mathbb{R}^{1}$ and for any initial data we have

$$
\left|x_{1}(t)^{2}-x_{2}(t)\right| \leq \exp (-2 a t)\left|x_{1}(0)^{2}-x_{2}(0)\right| .
$$

Thus, systems (20) and (22) have the same invariant exponentially attractive manifold $M$ on which almost all solutions of the first approximation system (22) have a positive characteristic exponent and all solutions of the original system (20) have negative characteristic exponents. The same result can be obtained for discrete systems $\left(20^{\prime}\right)$ and $\left(22^{\prime}\right)$.

The Perron effect occurs here on the whole manifold

$$
\left\{x_{3} \in \mathbb{R}^{1}, x_{2}=x_{1}^{2} \neq 0\right\} .
$$

\subsection{Stability criteria by the first approximation}

Represent the solutions of systems (1) and (1') in the Cauchy form. In the continuous case we have

$$
x(t)=X(t) x(0)+\int_{0}^{t} X(t) X(\tau)^{-1} f(\tau, x(\tau)) d \tau,
$$

and in the discrete one

$$
x(t)=X(t) x(0)+\sum_{\tau=0}^{t-1} X(t) X(\tau+1)^{-1} f(\tau, x(\tau)), t=1,2 \ldots
$$

Here $X(t)$ is a fundamental matrix of the linear part of the system.

Recall that by condition (2) the nonlinear part $f(t, x)$ of systems (1) and (1') in a certain neighborhood $\Omega(0)$ of the point $x=0$ satisfies the following condition

$$
|f(t, x)| \leq \kappa|x|^{\nu} \forall t \geq 0, \quad \forall x \in \Omega(0), \quad \kappa>0, \nu>1 .
$$

We now describe the most famous stability criteria for the solution $x(t) \equiv 0$ by the first approximation.

Consider the continuous case. We assume that there exists a number $C>0$ and a piecewise continuous function $p(t)$ such that for the Cauchy matrix $X(t) X(\tau)^{-1}$ the estimate

$$
\left|X(t) X(\tau)^{-1}\right| \leq C \exp \int_{\tau}^{t} p(s) d s, \quad \forall t \geq \tau \geq 0
$$

is valid.

Theorem 1 'Leonov, 2008] If condition (2) with $\nu=1$ and the inequality

$$
\lim _{t \rightarrow+\infty} \sup \frac{1}{t} \int_{0}^{t} p(s) d s+C \kappa<0
$$

are satisfied, then the solution $x(t) \equiv 0$ of system (1) is asymptotically Lyapunov stable. 
We now consider a discrete analog of this theorem. In the discrete case we assume that in place of inequality (26) we have

$$
\left|X(t) X(\tau)^{-1}\right| \leq C \prod_{s=\tau}^{t-1} p(s), \quad \forall t>\tau \geq 0,
$$

where $p(s)$ is a positive function.

Theorem 2 If condition (2) with $\nu=1$ and the inequality

$$
\lim _{t \rightarrow+\infty} \sup \frac{1}{t} \ln \prod_{s=0}^{t-1}(p(s)+C \kappa)<0
$$

are satisfied, then the solution $x(t) \equiv 0$ of system $\left(1^{\prime}\right)$ is asymptotically Lyapunov stable.

Corollary 1 For the first-order system the negativeness of characteristic exponent of the first approximation system implies the asymptotic stability of zero solution.

We now assume that for the Cauchy matrix $X(t) X(\tau)^{-1}$ the following estimate

$$
\left|X(t) X(\tau)^{-1}\right| \leq C \exp (-\alpha(t-\tau)+\gamma \tau), \quad \forall t \geq \tau \geq 0
$$

where $\alpha>0, \gamma \geq 0$, is satisfied.

Theorem $3{ }_{i}^{\prime}$ Chetaev, 1955, Malkin, 1966, Massera, 1957] Let condition (2) with sufficiently small $\kappa$ and condition (29) be valid. Then if the inequality

$$
(\nu-1) \alpha-\gamma>0
$$

holds, then the solution $x(t) \equiv 0$ is asymptotically Lyapunov stable.

Theorem 3 strengthens the well-known Lyapunov theorem on stability by the first approximation for regular systems |Lyapunov, 1892|.

\subsection{Stability criteria for the flow and cascade of solutions}

Consider a continuous system

$$
\frac{d x}{d t}=F(x, t), \quad x \in \mathbb{R}^{n}, \quad t \geq 0
$$

and the discrete one

$$
x(t+1)=F(t, x(t)), \quad x(t) \in \mathbb{R}^{n}, \quad t=0,1,2, \ldots,
$$

where $F(\cdot, \cdot)$ is a twice continuously differentiable vector-function.

Consider the linearizations of these systems along solutions with the initial data $y=x(0, y)$ from the open set $\Omega$, which is bounded in $\mathbb{R}^{n}$

$$
\begin{gathered}
\frac{d z}{d t}=A_{y}(t) z, \\
z(t+1)=A_{y}(t) z(t) .
\end{gathered}
$$

Here the matrix

$$
A_{y}(t)=\left.\frac{\partial F(x, t)}{\partial x}\right|_{x=x(t, y)}
$$


is Jacobian matrix of the vector-function $F(x, t)$ on the solution $x(t, y)$. Let $X(t, y)$ be a fundamental matrix of linear system and $X(0, y)=I_{n}$.

We assume that for the largest singular value $\alpha_{1}(t, y)$ of systems (32) and (32') for all $t$ the following estimate

$$
\alpha_{1}(t, y)<\alpha(t), \quad \forall y \in \Omega,
$$

where $\alpha(t)$ is a scalar function, is valid.

Theorem 4 'Leonov, 1998, Kuznetsov 85 Leonov, 2005² Suppose, the function $\alpha(t)$ is bounded on the interval $(0,+\infty)$. Then the flow (cascade) of solutions $x(t, y), y \in \Omega$, of systems (31) and (31') are Lyapunov stable.

If, in addition,

$$
\lim _{t \rightarrow+\infty} \alpha(t)=0
$$

then the flow (cascade) of solutions $x(t, y), y \in \Omega$, is asymptotically Lyapunov stable.

Corollary 2 The Perron effects are possible on the boundary of the stable by the first approximation solutions flow (cascade) only.

\section{Instability Criteria by the First Approximation}

\subsection{The Perron - Vinograd triangulation method}

One of basic procedures for analysis of instability is a reduction of the linear part of the system to the triangular form. In this case the Perron - Vinograd triangulation method for a linear system |Demidovich, 1967, Bylov et al., 1966, Coppel, 1978| turns out to be most effective. It will be described below.

Let

$$
Z(t)=\left(z_{1}(t), \ldots, z_{n}(t)\right)
$$

be a fundamental system of solutions of linear continuous system (3) or discrete system $\left(3^{\prime}\right)$.

We apply the Schmidt orthogonalization procedure to the solutions $z_{j}(t)$.

$$
\begin{aligned}
& v_{1}(t)=z_{1}(t) \\
& v_{2}(t)=z_{2}(t)-v_{1}(t)^{*} z_{2}(t) \frac{v_{1}(t)}{\left|v_{1}(t)\right|^{2}} \\
& \ldots \ldots \ldots \ldots \ldots \\
& v_{n}(t)=z_{n}(t)-v_{1}(t)^{*} z_{n}(t) \frac{v_{1}(t)}{\left|v_{1}(t)\right|^{2}}-\ldots-v_{n-1}(t)^{*} z_{n}(t) \frac{v_{n-1}(t)}{\left|v_{n-1}(t)\right|^{2}} .
\end{aligned}
$$

We proceed now to the description of the triangulation procedure of Perron - Vinograd.

Consider the unitary matrix

$$
U(t)=\left(\frac{v_{1}(t)}{\left|v_{1}(t)\right|}, \cdots, \frac{v_{n}(t)}{\left|v_{n}(t)\right|}\right),
$$

and make the change of variable: $z=U(t) w$ in the linear system. In the continuous case we obtain the system

$$
\frac{d w}{d t}=B(t) w
$$

where

$$
B(t)=U(t)^{-1} A(t) U(t)-U(t)^{-1} \dot{U}(t)
$$


and in the discrete case the system

$$
w(t+1)=B(t) w(t)
$$

where

$$
B(t)=U(t+1)^{-1} A(t) U(t) .
$$

The unitarity of the matrix $U(t)$ implies that for the columns $w(t)$ of the fundamental matrix

$$
W(t)=\left(w_{1}(t), \ldots, w_{n}(t)\right)=U(t)^{*} Z(t),
$$

the relations $\left|w_{j}(t)\right|=\left|z_{j}(t)\right|$ are satisfied.

By (34) we obtain that the matrix $W(t)$ has the upper triangular form with the diagonal elements $\left|v_{1}(t)\right|, . .,\left|v_{n}(t)\right|$, namely

$$
W(t)=\left(\begin{array}{ccc}
\left|v_{1}(t)\right| & \cdots & \\
& \ddots & \vdots \\
0 & & \left|v_{n}(t)\right|
\end{array}\right) .
$$

From the fact that $W(t)$ is an upper triangular matrix it follows that $W(t)^{-1}, \dot{W}(t)$ are also upper triangular matrices. Hence $B(t)$ is an upper triangular matrix with the diagonal elements $b_{1}(t), . ., b_{n}(t)$ :

$$
B(t)=\left(\begin{array}{ccc}
b_{1}(t) & \cdots & \\
& \ddots & \vdots \\
0 & & b_{n}(t)
\end{array}\right),
$$

where in the continuous case $b_{i}(t)=\left(\ln \left|v_{i}(t)\right|\right)^{\bullet}$ and in the discrete one

$$
b_{i}(t)=\frac{\left|v_{i}(t+1)\right|}{\left|v_{i}(t)\right|}
$$

Thus, we proved the following

Theorem 5 (Perron triangulation [Demidovich, 1967, Kuznetsov \& Leonov, 200517) By means of the unitary transformation $z=U(t) w$ the linear system can be reduced to the linear system with the upper triangular matrix $B(t)$.

Note that if $|A(t)|$ is bounded for $t \geq 0$, then $|B(t)|,|U(t)|$, and $|\dot{U}(t)|$ are also bounded for $t \geq 0$. If in the discrete case, in addition, $\left|A(t)^{-1}\right|$ is bounded for $t \geq 0$, then $\left|B(t)^{-1}\right|$ is also bounded for $t \geq 0$.

Define the vector $z_{i}^{\prime}=z_{i}-v_{i}$. Then the vector $z_{i}^{\prime}$ is orthogonal to the vector $v_{i}$, where $i \geq 2$. Consider the angle included between the vectors $z_{i}$ and $z_{i}^{\prime}$. Note that from definition of the angle included between the vectors we have $\angle\left(z_{i}, z_{i}^{\prime}\right) \leq \pi$. In this case the following relation

$$
\left|v_{i}\right|=\left|z_{i}\right| \sin \left(\angle\left(z_{i}, z_{i}^{\prime}\right)\right) \quad i \geq 2
$$

is valid.

By (40) from (37) and (38) we have

$$
|\operatorname{det} Z(t)|=|\operatorname{det} U(t)| \prod_{i=1}^{n}\left|v_{i}\right|=\prod_{i=1}^{n}\left|z_{i}\right| \prod_{k=2}^{n}\left|\sin \left(\angle\left(z_{k}, z_{k}^{\prime}\right)\right)\right| \text {. }
$$

With the help of this relation in |Vinograd, 1954| the following criterion of system regularity was obtained. 
Theorem 6 'Vinograd, 1954]

Consider a linear system with bounded coefficients and its certain fundamental system of solutions $Z(t)=$ $\left(z_{1}(t), \ldots, z_{n}(t)\right)$. Let there exist the exact characteristic exponents of $\left|z_{i}(t)\right|$

$$
\lim _{t \rightarrow+\infty} \frac{1}{t} \ln \left|z_{i}(t)\right| \quad i=1, \ldots, n
$$

and let there exist and be equal to zero the exact characteristic exponents of sines of the angles $\angle\left(z_{i}, z_{i}^{\prime}\right)$

$$
\lim _{t \rightarrow+\infty} \frac{1}{t} \ln \left|\sin \left(\angle\left(z_{i}, z_{i}^{\prime}\right)\right)\right|=0 \quad i=2, \ldots, n
$$

Then the linear system is regular and $Z(t)$ is a normal system of solutions.

Conversely, if the linear system is regular and $Z(t)$ is a normal system of solutions, then (41) and (42) are satisfied.

\subsection{Instability criterion by Krasovsky}

Consider instability by Krasovsky for the solution $x(t) \equiv 0$ of continuous system (1) and of discrete system $\left(1^{\prime}\right)$.

In the continuous case the following theorem strengthens the result, obtained in |Leonov, 2002 ${ }^{1}$, Leonov, 2004|. In the discrete case this result is reduced to that, obtained in $\mid$ Kuznetsov \& Leonov, $2005^{1} \mid$.

Theorem 7 If the relation

$$
\sup _{1 \leq k \leq n} \lim _{t \rightarrow+\infty} \inf \left[\frac{1}{\ln t}\left(\ln |\operatorname{det} Z(t)|-\sum_{j \neq k} \ln \left|z_{j}(t)\right|\right)\right]>1
$$

is satisfied, then the solution $x(t) \equiv 0$ is unstable by Krasovsky.

\section{Remark, concerning the method for the proof of theorem.}

Assuming that the zero solution of the considered system is Lyapunov stable and using the same reasoning as in the case of stability by Krasovsky, we need to prove in the continuous case the following inequality

$$
y_{n}(0)+\int_{0}^{+\infty} \frac{\left|v_{n}(0)\right|}{\left|v_{n}(s)\right|} g(s, y(s)) d s \neq 0
$$

While the above inequality is easily proved in the case of stability by Krasovsky, this becomes an intractable problem in the case of stability by Lyapunov.

A scheme similar to that, considered above for reducing the problem to one scalar equation, was used by N.G. Chetaev $[1990 ; 1948]$ to obtain instability criteria. In the scheme, suggested by N.G. Chetaev for proving inequality (44), a similar difficulty occurs. Therefore, at present, Chetaev's technique permits us to obtain the criteria of instability by Krasovsky only.

The method to obtain the criteria of instability by Lyapunov invites further development. Such development under certain additional restrictions will be presented in Theorem 9.

Condition (43) of Theorem 7 is satisfied if the following inequality

$$
\Lambda-\Gamma>0
$$

is valid.

Here $\Lambda$ is the largest characteristic exponent, $\Gamma$ is the irregularity coefficient. 
The condition of instability by Krasovsky (45) was obtained under the additional condition of the analyticity of the function $f(t, x)$ by N.G. Chetaev $[1990 ; 1948]$

Recall here stability condition (30) of Theorem 3, which can be represented as

$$
(\nu-1) \Lambda+\Gamma<0 .
$$

\section{Theorem 8 If}

$$
\Lambda<\frac{-\Gamma}{(\nu-1)},
$$

then the solution $x(t) \equiv 0$ is Krasovsky stable and if

$$
\Lambda>\Gamma
$$

then the solution $x(t) \equiv 0$ is Krasovsky unstable.

For regular systems (the case $\Gamma=0$ ), Theorem 8 gives a complete solution of the problem of stability by Krasovsky in the noncritical case $(\Lambda \neq 0)$.

Note that for system (22) the relation $\Gamma=\Lambda+2 a+1$ holds. Therefore for system (22) condition (45) is untrue.

\subsection{Instability criterion by Lyapunov}

Consider now the Lyapunov instability of the solution $x(t) \equiv 0$ of multidimensional continuous system (1) and of discrete system (1').

Theorem 9 [Leonov, 2004, Kuznetsov 83 Leonov, 2005'] Let for certain values $C>0, \beta>0, \alpha_{1}, \ldots, \alpha_{n-1}\left(\alpha_{j}<\right.$ $\beta$ for $j=1, \ldots, n-1)$ the following conditions hold:

1)

$$
\begin{gathered}
\left|z_{j}(t)\right| \leq C \exp \left(\alpha_{j}(t-\tau)\right)\left|z_{j}(\tau)\right|, \\
\forall t \geq \tau \geq 0, \quad j=1, \ldots, n-1,
\end{gathered}
$$

2)

$$
\frac{1}{(t-\tau)} \ln |\operatorname{det} Z(t)|>\beta+\sum_{j=1}^{n-1} \alpha_{j}, \quad \forall t \geq \tau \geq 0,
$$

and, if $n>2$,

3)

$$
\prod_{j=1}^{n}\left|z_{j}(t)\right| \leq C|\operatorname{det} Z(t)|, \quad \forall t \geq 0 .
$$

Then the zero solution of the system considered is Lyapunov unstable. 


\subsection{Instability criterion for the flow and cascade solutions}

The problem arises naturally as to the weakening of instability conditions, which are due to Theorems 7 and

9. However the Perron effects impose restrictions on such weakening.

We now turn to continuous and discrete systems (31) and (31'), respectively.

Suppose, for a certain vector-function $\xi(t)$ the following relations

$$
|\xi(t)|=1, \quad \inf _{y \in \Omega}|X(t, y) \xi(t)| \geq \alpha(t), \quad \forall t \geq t_{0}
$$

hold.

Theorem 10 [Leonov, 1998, Kuznetsov \& Leonov, 20051] Let for the function $\alpha(t)$ the following condition

$$
\lim _{t \rightarrow+\infty} \sup \alpha(t)=+\infty
$$

be satisfied.

Then the flow (cascade) of solutions $x(t, y), y \in \Omega$ is Lyapunov unstable.

Now we consider the hypotheses of Theorem 10.

The hypotheses of Theorem 10 is, in essence, the requirement that, at least, one Lyapunov exponent of the linearizations of the flow of solutions with the initial data from $\Omega$ is positive under the condition that the "unstable directions $\xi(t)$ " (or unstable manifolds) of these solutions depend continuously on the initial data $x_{0}$. Actually, if this property holds, then, regarding (if necessary) the domain $\Omega$ as the union of the domains $\Omega_{i}$, of arbitrary small diameter, on which conditions (50) and (51) are valid, we obtain the Lyapunov instability of the whole flow of solutions with the initial data from $\Omega$.

\section{Conclusion}

We summarize the investigations of stability by the first approximation.

Theorems 4 and 10 give a complete solution for the problem on the flows and cascade of solutions in the noncritical case when for small variations of the initial data of the original system the system of the first approximation preserves its stability (or instability in the certain "direction" $\xi(t)$ ).

Thus, the classical problem of stability by the first approximation of nonstationary motions is completely proved in the general case |Malkin, 1966|.

The Perron effects are possible only on the boundaries of the flows that are either stable or unstable by the first approximation. From this point of view we have here the special case.

In the general case, the progress became possible since the theorem on finite increments permits us to reduce the estimate of the difference between perturbed and unperturbed solutions to the analysis of the first approximation system, linearized along a certain "third" solution of the original system. Such an approach makes the proof of the theorem "almost obvious".

Thus, the difficulties, arising in studying the individual solutions, are connected to the fact that these solutions can be situated on the boundaries of the flows that are stable (or unstable) by the first approximation. In this case a special situation occurs which requires the development of finer (and, naturally, more complicated) tools for investigation. Such methods of investigation of the individual solutions are given in the present study. 


\section{References}

[Andrianova, 1998] Adrianova, L.Ya. [1998] Introduction to Linear systems of Differential Equations (American Mathematical Society, Providence, Rhode Island).

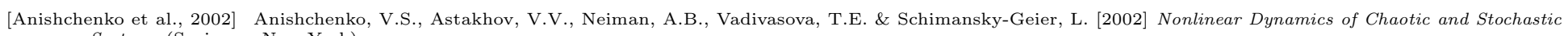
Systems (Springer, New York).

[Bacciotti \& Rosier, 2005] Bacciotti, A. \& Rosier, L. [2005] Liapunov functions and stability in control theory, 2nd Edition (Springer).

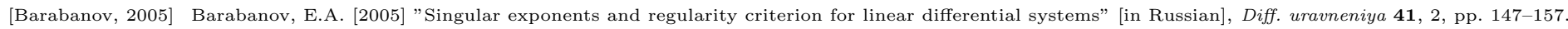

[Bellman, 1953] Bellman, R. [1953] Stability theory of differential equations (New York, McGraw-Hill).

[Bellman \& Cooke, 1963] Bellman, R. \& Cooke, K.L. [1963] Differential-difference equations (Academic Press, New York-London).

[Boichenko et al., 2005] Boichenko, V.A., Leonov, G.A. \& Reitmann, V. [2005] Dimension Theory for Ordinary Differential Equations (Teubner, Stuttgart).

[Bromberg, 1986] Bromberg, V.P. [1986] Matrix method in the theory of relay and pulse regulation [in Russian] (Nauka, Moscow).

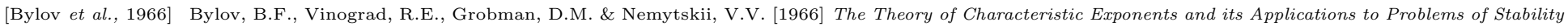
[in Russian] (Nauka, Moscow).

[Cesari, 1959] Cesari, L. [1959] Asymptotic Behavior and Stability Problems in Ordinary Differential Equations (Springer, Berlin).

[Chetaev, 1990] Chetaev, N.G. [1990] Stability of Motion [in Russian] (Nauka, Moscow).

[Chetaev, 1955] Chetaev, N.G. [1955] Stability of motion [in Russian] (Gostekhizdat, Moscow).

[Chetaev, 1948] Chetaev, N.G. [1948] "Some problems of stability and instability for nonregular systems" [in Russian], Prikl. Math. i Mech. 12, 6, pp. 639-642.

[Coppel, 1978] Coppel, W.A. [1978] Dichotomies in Stability Theory (Springer-Verlag).

[Coppel, 1965] Coppel, W.A. [1965] Stability and asymptotic behavior of differential equations (D.C. Heath, Boston).

[Davies \& James, 1966] Davies, T.V. \& James, E.M. [1966] Nonlinear differential equations (Addison-Wesley).

[Demidovich, 1969] Demidovich, V.B. [1969] "Stability criterion for difference equations" [in Russian], Diff. uravneniya 5, 7, pp. 1247-1255.

[Demidovich, 1967] Demidovich, B.P. [1967] Lectures on mathematical theory of stability [in Russian] (Nauka, Moskva).

[Filippov, 1988] Filippov, A.F. [1988] Differential Equations with Discontinuous Right Hand Sides (Kluwer, Dordrecht).

[Gayschun, 2001] Gayschun, I.V. [2001] Systems with discrete time [in Russian] (Inst. matemat. RAN Belarusi, Minsk).

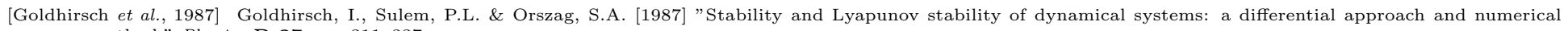
method," Physica D 27, pp. 311-337.

[Halanay, 1966] Halanay, A. [1966] Differential Equation (Academic Press, New York-London).

[Hahn, 1967] Hahn, W. [1967] Stability of motion (Springer-Verlag, Berlin-Heidelberg).

[Hartman, 1984] Hartman, P. [1984] Ordinary differential equations, Second edition (BirkHauser).

[Heagy \& Hammel, 1994] Heagy, J.F. \& Hammel, S.M. [1994] "The birth of strange nonchaotic attractors," Physica D 70, N1,2, pp. 140-153.

[Katok \& Hasselblat, 1995] Katok, A. \& Hasselblatt, B. [1995] Introduction to the modern theory of dynamical systems (Cambridge univ. press).

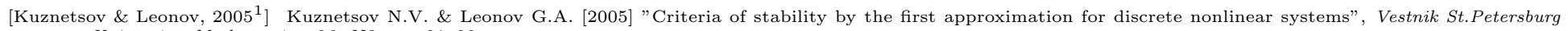
University. Mathematics. 38, N3, pp. 21-30.

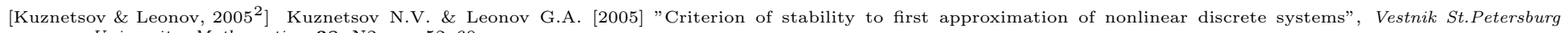
University. Mathematics. 38, N2, pp. 52-60.

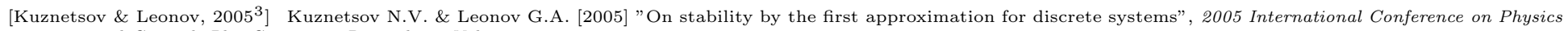
and Control, PhysCon 2005, Proceedings Volume 2005, 2005, art. num. 1514053, pp. 596-599.

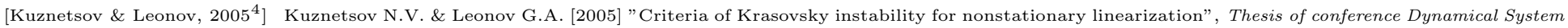
Modeling And Stability Investigation, p. 72 .

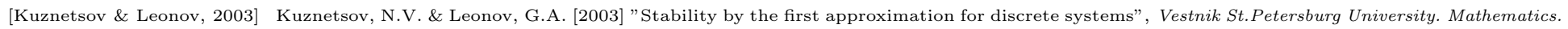
36, 1, pp. 21-27.

[Kuznetsov \& Leonov, 2001] Kuznetsov, N.V. \& Leonov, G.A. [2001] "Counterexample of Perron in the discrete case", Izv. RAEN, Diff. Uravn., 5, p. 71.

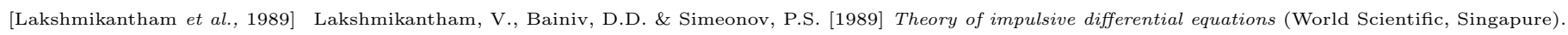

[LaSalle, 1980] LaSalle, J.P. [1980] The Stability and Control of Discrete Processes (Springer-Verlag).

[Leonov, 2008] Leonov, G.A. [2008] Strange attractors and classical stability theory [in English] (St. Petersburg University Press, Russia).

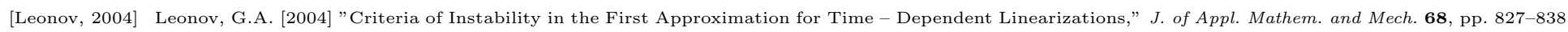

[Leonov, 2003] Leonov, G.A. [2003] "A Modification of Perron's Counterexample," Differential Equations 39, 11, pp. 1651-1652

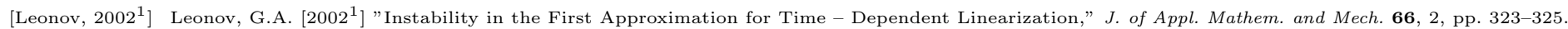


[Leonov, $2002^{2}$ ] Leonov, G.A. [2002 ${ }^{2}$ ] "Strange attractors and the classical theory of motion stability" [in Russian], Uspekhi mekhaniki 1, 3, pp. 3-42.

[Leonov, 2001 ${ }^{1}$ ] Leonov, G.A. [2001] Mathematical Problems of Control Theory, An Introduction (World Scientific, Singapore)

[Leonov, 20012] Leonov, G.A. [2001] "Lyapunov Dimension Formulas for Henon and Lorenz Attractors," St.Petersburg Mathem. J. 13, 3, pp. 453-464.

[Leonov, 1998] Leonov, G.A. [1998] "On Stability in the First Approximation," J. of Appl. Mathem. and Mech. 62, 4, pp. 511-517.

[Luca \& Vleck, 2000] Luca, D. \& Van Vleck, E. [2000] "Lyapunov and other spectra: a survey," SIAM J. Numer. Anal..

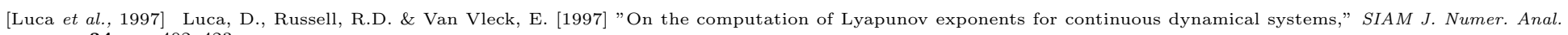
34, pp. 402-423.

[Lyapunov, 1892] Lyapunov, A.M. [1892] Stability of Motion (Academic Press, New York).

[Malkin, 1966] Malkin I.G., Theory of motion stability [in Russian] (Nauka, Moscow).

[Massera, 1957] Massera, J.L. [1958] "Contributions to Stability Theory," Ann. Math., 64, pp. 182-206; 68, p. 202.

[Maxwell, 1868] Maxwell, J. [1868] "On the governors," Proceedings of the Royal Society, 270, 16.

[Maxwell et al., 1949] Maxwell, D.K., Vyshnegradskii, I.A. \& Stodola, A. [1949] Automatic Control Theory, Linearized Problems (AN SSSR, Moscow).

[Moon, 1987] Moon, F. [1987] Chaotic Vibrations (John Wiley, New York).

[Neimark \& Landa, 1992] Neimark, Yu.I. \& Landa, P.S. [1992] Stochastic and Chaotic Oscillations (Kluwer, Dordrecht).

[Perron, 1930] Perron, O. [1930] "Die Stabilitatsfrage bei Differentialgleichungen," Mathematische Zeitschrift bd. 32, 5, pp. 702-728.

[Persidskii, 1947] Persidskii, K.P. [1947] "Characteristic numbers of differential equations" [in Russian], Izv. AN Kazakhskoi SSR, ser.mat.i mekh., 1.

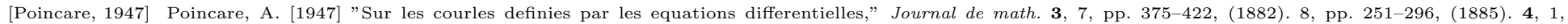
pp. 167-244, (1886). 2, pp. 151-217.

[Russel et al., 1980] Russel, D.A., Hanson, J.D. \& Ott, E. [1980] "Dimension of Strange Attractors," Phys. Rev. Lett. 45, 14, pp. 1175-1178.

[Ryabov, 2002] Ryabov, V.B. [2002] "Using Lyapunov exponents to predict the onset of chaos in nonlinear oscillators," Physical Review E 66, pp. 16214-16231.

[Sansone \& Conti 1964] Sansone, G. \& Conti, R. [1964] Non-linear differential equations (Pergamon Press).

[Schuster, 1984] Schuster, H.G. [1984] Deterministic Chaos (Physik - Verlag, Weinheim).

[Temam, 1988] Temam, R. [1988] Infinite-Dimensional Dynamical Systems in Mechanics and Physics (Springer-Verlag, New York).

[Vinograd, 1954] Vinograd, R.E. [1954] Uspekhi matematicheskih nauk 9, 2, pp. 129-136 [in Russian].

[Vyshnegradskii, 1877] Vyshnegradskii, I.A. [1877] "Direct-acting regulators" [in Russian], Izv. Sankt-Peterburgskogo tekhnologicheskogo instituta, pp. 21-62.

[Zorich, 1984] Zorich, V.A. [1984] Mathematical analysis. Parts I, II [in Russian] (Nauka, Moscow).

[Wasow, 1965] Wasow, W. [1965] Asymptotic expansions for ordinary differential equations (Interscience publ., New York).

[Willems, 1970] Willems, J.L. [1970] Stability theory of dynamical systems (John Willey and Sons, Inc., New York).

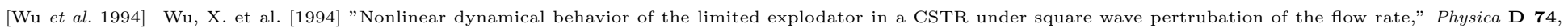
1,2 , pp. $74-89$.

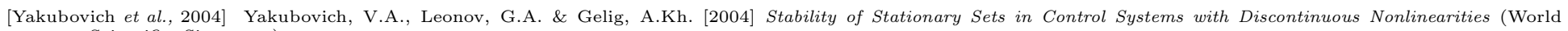
Scientific, Singapore).

[Yoshizawa, 1966] Yoshizawa, T. [1966] Stability theory by Liapunov's second method (The Mathematical Society of Japan, Tokio). 